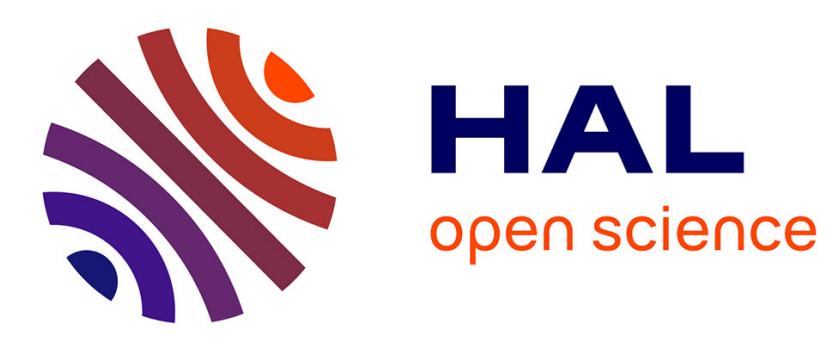

\title{
Sur le problème des phases dans le couplage entre le spin électronique et la rotation moléculaire
}

\author{
C. Di Lauro, F. Lattanzi
}

\section{To cite this version:}

C. Di Lauro, F. Lattanzi. Sur le problème des phases dans le couplage entre le spin électronique et la rotation moléculaire. Journal de Physique, 1979, 40 (2), pp.129-136. 10.1051/jphys:01979004002012900 . jpa-00208891

\section{HAL Id: jpa-00208891 https://hal.science/jpa-00208891}

Submitted on 1 Jan 1979

HAL is a multi-disciplinary open access archive for the deposit and dissemination of scientific research documents, whether they are published or not. The documents may come from teaching and research institutions in France or abroad, or from public or private research centers.
L'archive ouverte pluridisciplinaire HAL, est destinée au dépôt et à la diffusion de documents scientifiques de niveau recherche, publiés ou non, émanant des établissements d'enseignement et de recherche français ou étrangers, des laboratoires publics ou privés. 


\title{
Sur le problème des phases dans le couplage entre le spin électronique et la rotation moléculaire
}

\author{
C. di Lauro et F. Lattanzi \\ Dipartimento di Fisica, Università della Calabria, 87030 \\ Arcavacata di Rende (Cosenza), Italie
}

(Reçu le 21 avril 1978, révisé le 16 octobre 1978, accepté le 26 octobre 1978)

\begin{abstract}
Résumé. - On définit des angles déterminés par les phases đes fonctions d'onde des représentations couplée et découplée dans le couplage entre la rotation moléculaire et le spin électronique. Puisque les coefficients de ClebschGordan des tableaux standard [1-3] correspondent à des valeurs particulières de ces angles, c'est-à-dire à des relations de phases fixées dans les deux représentations (phases et angles standard), on donne une relation permettant de les modifier en vue de les appliquer à des problèmes où l'on fait des choix de phases différents. Examinant les différentes conventions suivies dans la littérature, nous discutons aussi des exemples d'applications à la spectroscopie ro-vibronique. Dans l'appendice I on détermine la valeur des C. G. à utiliser dans l'expression des éléments de matrice des composantes sphériques de tenseurs irréductibles obtenue par le théorème de Wigner-Eckart, dans le cas où on ne suit pas les conventions standard. Certaines propriétés des opérateurs cosinus directeurs sont examinées dans l'appendice II.
\end{abstract}

\begin{abstract}
Angles related to the relative phases of the wavefunctions in the coupled and uncoupled representations of molecular rotation and electron spin are defined. The dependence of the analytical form of the Clebsch-Gordan coefficients and their recurrence relations on the relative phases in both representations is discussed, and a general expression suited to properly modify the C. G. coefficients of the standard tables [1-3] when unconventional phases are adopted is given. Practical cases in the literature of applications to problems of ro-vibronic spectroscopy are discussed. A rule to determine the values of the C. G. coefficients occurring in the expression of the matrix elements of the spherical components of irreducible tensors through the Wigner-Eckart theorem, when unconventional phases are adopted, is finally given in appendix I. Some properties of the direction cosine operators are examined in appendix II.
\end{abstract}

Introduction. - Une lecture rapide de la littérature concernant l'analyse rotationnelle des spectres électroniques des molécules polyatomiques avec spin électronique, dans l'espace libre comme sous l'effet de champs externes, montre aisément qu'on utilise souvent des fonctions de base ayant des phases non conventionnelles. Alors les coefficients de ClebschGordan (C. G.) des tableaux standard [1-3] ne sont plus applicables aux procédés de couplage des moments cinétiques qu'on rencontre dans ces cas. Dans ce domaine de la spectroscopie, différents auteurs ont adopté différentes conventions de phases, provoquant ainsi des complications ultérieures. De plus, dans les problèmes concernant le couplage entre la rotation de la molécule $(\mathbf{N})$ et le spin électronique (S) donnant la résultante $\mathbf{J}$, on peut définir simultanément pour $\mathbf{N}$ et $\mathbf{J}$ les deux projections $Z$ et $z$, dans les systèmes d'axes liés au laboratoire (référentiel fixe) et à la molécule (référentiel mobile) respective- ment, au moyen des nombres quantiques $M_{N}$ et $k$ pour $\mathbf{N}$ et $M_{J}$ et $P$ pour $\mathbf{J}$. On peut alors obtenir la représentation couplée $\left|N k J M_{J} S\right\rangle$ par le couplage $\mathbf{J}=\mathbf{N}+\mathbf{S}$ dans le référentiel fixe et par le couplage anormal $\mathbf{N}=\mathbf{J}-\mathbf{S}$ dans le référentiel mobile (Van Vleck [4]), et on doit faire très attention à engendrer cette Peprésentation avec les mêmes phases dans les deux cas. Le but de cet article est donc surtout de donner des règles simples et pratiques permettant de modifier aisément les C. G. des tableaux standard, qui correspondent à des relations de phases fixées dans les représentations couplée et découplée, lorsqu'il faut les appliquer à des problèmes où les conventions sur les phases sont déterminées par des choix différents.

Un traitement général pouvant être appliqué à ces problèmes a été donné par Fémenias et Athénour [5]. Des modalités d'application de l'algèbre standard des moments cinétiques dans le référentiel mobile, et des 
considérations sur les conventions de phases ont été aussi données par Freed [6] et par Brown et Howard [7]. Après un paragraphe consacré à préciser les définitions et notations adoptées dans cet article, nous montrons dans la section 1 de quelle façon les valeurs des C. G. dépendent des relations de phases choisies dans les deux représentations mentionnées, en donnant une attention particulière aux problèmes de la spectroscopie ro-vibronique. Dans ta section 2 nous discutons des exemples de la littérature.

Notation ET DÉFinitions. - Dans cet article les valeurs des moments cinétiques sont toujours données en unités $\hbar$.

Nous écrivons la relation de couplage entre les moments cinétiques dans la forme générale $\mathbf{j}=\mathbf{j}_{1}+\mathbf{j}_{2}$ : la notation adoptée pour les nombres quantiques de ces opérateurs, et la correspondance avec les nombres quantiques qui se rapportent aux opérateurs $\mathbf{N}$ (rotation moléculaire), $\mathbf{S}$ (spin électronique) et $\mathbf{J}$ (résultante) sont données dans le tableau. Il faut remarquer

Tableau I. - Correspondance des nombres quantiques entre la notation générale de couplage $\mathbf{j}=\mathbf{j}_{1}+\mathbf{j}_{2}$ et les deux schémas de couplage spin électronique-rotation moléculaire.

\begin{tabular}{cccccccccc} 
& \multicolumn{1}{c}{ Notation générale $: \mathbf{j}=\mathbf{j}_{1}+\mathbf{j}_{2}$} \\
Schéma de couplage & \multicolumn{1}{c}{ Nombres quantiques } \\
& $j_{1}$ & $j_{2}$ & $j$ & $m_{1}$ & $n_{1}$ & $m_{2}$ & $m$ & $n$ \\
$\mathbf{J}=\mathbf{N}+\mathbf{S}$ & $N$ & $S$ & $J$ & $M_{N}$ & $k$ & $M_{S}$ & $M_{J}$ & $P$ \\
$($ Référentiel fixe) & & & & & & & & \\
$\quad \begin{array}{c}\mathbf{J}-\mathbf{S} \\
\text { (Référentiel mobile) }\end{array}$ & $J$ & $S$ & $N$ & $P$ & $M_{J}$ & $-\Sigma$ & $k$ & $M_{N}$ \\
& & & & & & & &
\end{tabular}

$M_{N}, M_{S}, M_{J}$ se rapportent aux composantes quantisées dans le référentiel fixe, et $k, \Sigma, P$, aux composantes dans le référentiel mobile.

Meaning of the quantum numbers involved in the general coupling relation $j=j_{1}+j_{2}$ for the two coupling schemes between electron spin and molecular rotation.

que dans le tableau deux nombres quantiques de projection sont donnés pour les opérateurs $\mathbf{j}$ et $\mathbf{j}_{1}$, qui correspondent à $\mathbf{N}$ et $\mathbf{J}$ dont les composantes $z$ dans les deux référentiels fixe et mobile commutent entre elles.

Les représentations découplée et couplée dans notre notation sont formées respectivement par les fonctions suivantes :

$$
\begin{aligned}
& \left|j_{1} m_{1} n_{1} j_{2} m_{2}\right\rangle=\left|j_{1} m_{1} n_{1}\right\rangle\left|j_{2} m_{2}\right\rangle \\
& \left|j m j_{1} n_{1} j_{2}\right\rangle=\sum_{\substack{m_{2} \\
m_{1}=m-m_{2}}} \times \\
& \quad \times\left\langle j_{1} m_{1} n_{1} j_{2} m_{2} \mid j m j_{1} n_{1} j_{2}\right\rangle\left|j_{1} m_{1} n_{1} j_{2} m_{2}\right\rangle .
\end{aligned}
$$

Dans la notation de cet article, les indices supérieurs + et - identifient les opérateurs qui modifient les nombres quantiques de projection de +1 et -1 respectivement, tandis que les indices + et - inférieurs caractérisent les combinaisons des composantes cartésiennes du type $x \pm i y$. Alors dans le référentiel fixe $\mathbf{j}^{ \pm}=\mathbf{j}_{ \pm}=\mathbf{j}_{X} \pm i \mathbf{j}_{Y}, \quad \mathbf{j}_{1}^{ \pm}=\mathbf{j}_{1 \pm}=\mathbf{j}_{1 X} \pm i \mathbf{j}_{1 Y}$, $\mathbf{j}_{2}^{ \pm}=\mathbf{j}_{2 \pm}=\mathbf{j}_{2 X} \pm i \mathbf{j}_{2 Y}$. Mais dans le référentiel mobile, à cause des relations de communications anormales [4] de $\mathbf{j}$ et $\mathbf{j}_{\mathbf{1}}$ (c'est-à-dire $\mathbf{N}$ et $\mathbf{J}$ ), nous avons

$$
\begin{aligned}
& \mathbf{j}^{ \pm}=\mathbf{j}_{\mp}=\mathbf{j}_{x} \mp i \mathbf{j}_{y}, \quad \mathbf{j}_{1}^{ \pm}=\mathbf{j}_{1 \mp}=\mathbf{j}_{1 x} \mp i \mathbf{j}_{1 y}, \\
& \mathbf{j}_{2}^{ \pm}=\mathbf{j}_{2 \pm}=\mathbf{j}_{2 x} \pm i \mathbf{j}_{2 y} .
\end{aligned}
$$

Quand il est nécessaire de les distinguer, les composantes dans le référentiel fixe et mobile sont identifiées respectivement par des indices majuscules et minuscules comme ci-dessus. Dans tous les autres cas, nos indices sont minuscules.

Enfin, nous représentons les C. G. des tableaux standard par les symboles $C\left(j_{1} j_{2} j ; m_{1} m_{2} m\right)$ de Rose [2] et les C. G. avec phases générales par les brackets de Dirac $\left\langle j_{1} m_{1} j_{2} m_{2} \mid j m j_{1} j_{2}\right\rangle$.

1. Phases et coefficients de Clebsch-Gordan. - 1.1 RePRÉSENTATION DÉCOUPLÉE. - Les phases relatives des fonctions d'onde de la représentation découplée par rapport aux variations des nombres quantiques de projections sont déterminées par les éléments de matrice

$$
\begin{gathered}
\left\langle j_{k} m_{k} \pm 1\left|\mathbf{j}_{k}^{ \pm}\right| j_{k} m_{k}\right\rangle=\exp \left( \pm i \delta_{k}\right) \times \\
\times\left[j_{k}\left(j_{k}+1\right)-m_{k}\left(m_{k} \pm 1\right)\right]^{1 / 2}
\end{gathered}
$$

En écrivant la relation de couplage $\mathbf{J}=\mathbf{N}+\mathbf{S}$ ou $\mathbf{N}=\mathbf{J}-\mathbf{S}$ sous la forme générale $\mathbf{j}=\mathbf{j}_{1}+\mathbf{j}_{2}$, on peut définir dans la représentation découplée les deux composantes $Z$ et $z$ de $\mathbf{j}_{1}$; il faut alors choisir trois angles de phases $\delta_{1}, \delta_{1}^{\prime}$, et $\delta_{2}$ par rapport aux nombres quantiques de projection. L'angle $\delta_{1}$ se rapporte à la projection $m_{1}$ de $\mathbf{j}_{1}$ sur laquelle il faut sommer dans l'opération de couplage, c'est-à-dire $M_{N}$ dans le couplage $\mathbf{J}=\mathbf{N}+\mathbf{S}$ et $P$ dans le couplage $\mathbf{N}=\mathbf{J}-\mathbf{S}$, et $\delta_{1}^{\prime}$ se rapporte à l'autre projection $n_{1}$, c'est-à-dire $k$ et $M_{J}$ dans les deux cas mentionnés. Les relations entre les C. G. d'une même ligne des tableaux de C. G. sont déterminées par $\delta_{1}$ et $\delta_{2}$. Rose [2] a obtenu une relation entre les C. G. d'une même ligne, c'est-à-dire qui correspondent aux valeurs successives de $m_{2}$, par un procédé utilisant les opérateurs $\mathbf{j}_{1}^{ \pm}$et $\mathbf{j}_{2}^{ \pm}$, avec le choix standard $\delta_{1}=\delta_{2}=0$. Suivant le même procédé avec des valeurs de $\delta_{1}$ et $\delta_{2}$ arbitraires, on obtient : 


$$
\begin{aligned}
& {\left[j(j+1)-j_{1}\left(j_{1}+1\right)-j_{2}\left(j_{2}+1\right)-2 m_{2}\left(m-m_{2}\right)\right]\left\langle j_{1} m-m_{2} j_{2} m_{2} \mid j m j_{1} j_{2}\right\rangle=} \\
& \quad=\exp \left[i\left(\delta_{1}-\delta_{2}\right)\right]\left\{\left[j_{1}\left(j_{1}+1\right)-\left(m-m_{2}-1\right)\left(m-m_{2}\right)\right]\left[j_{2}\left(j_{2}+1\right)-\left(m_{2}+1\right) m_{2}\right]\right\}^{1 / 2} \\
& \quad \times\left\langle j_{1} m-m_{2}-1 j_{2} m_{2}+1 \mid j m j_{1} j_{2}\right\rangle+\exp \left[-i\left(\delta_{1}-\delta_{2}\right)\right] \\
& \quad \times\left\{\left[j_{1}\left(j_{1}+1\right)-\left(m-m_{2}+1\right)\left(m-m_{2}\right)\right]\left[j_{2}\left(j_{2}+1\right)-\left(m_{2}-1\right) m_{2}\right]\right\}^{1 / 2} \\
& \quad \times\left\langle j_{1} m-m_{2}+1 j_{2} m_{2}-1 \mid j m j_{1} j_{2}\right\rangle .
\end{aligned}
$$

C'est une relation le long des lignes des tableaux de C. G. Avec le choix standard $\delta_{1}=\delta_{2}=0$, et dans tous les cas où $\delta_{1}=\delta_{2}$, on obtient la relation (3.27) de Rose [2]. Il est donc évident que, avec des valeurs $\delta_{1}$ et $\delta_{2}$ arbitraires dans la représentation découplée, il faut multiplier les C. G. standard par

$\exp \left[-i\left(\delta_{1}-\delta_{2}\right) m_{2}\right]$.

Lorsque $j_{2}$, c'est-à-dire $S$ dans notre cas, reste inchangé, il faudra définir seulement un angle de phase $\eta_{1}$ par rapport aux variations de $j_{1}$. Il est commode de définir cet angle par les éléments de matrice des opérateurs cosinus directeurs entre les axes cartésiens des deux référentiels fixe et mobile, en raison de leur importance dans les calculs d'intensités rotationnelles. Les propriétés des opérateurs cosinus directeurs sont résumées dans l'appendice II, et on voit que le choix des phases des fonctions $\left|j_{1} m_{1} n_{1}\right\rangle$ par rapport à $j_{1}$ détermine la valeur des éléments de matrice réduits $\left\langle j_{1}^{\prime}\|\lambda\| j_{1}\right\rangle$. Shaffer et Louck [8] ont calculé les éléments de matrice des opérateurs $\lambda_{\mu \nu}$ et, en tenant compte de notre expression (II.1) et en explicitant le facteur de phase par rapport à $j_{1}$, on obtient :

$$
\begin{gathered}
\left\langle j_{1}^{\prime}\|\lambda\| j_{1}\right\rangle=\left[\exp i \eta_{1}\left(j_{1}^{\prime}-j_{1}\right)\right] \times \\
\times\left[\left(2 j_{1}+1\right) /\left(2 j_{1}^{\prime}+1\right)\right]^{1 / 2} .
\end{gathered}
$$

Notre expression (II.1) correspond à l'équation (16) de Shaffer et Louck [8], qui suivent la convention $\delta_{1}=\delta_{1}^{\prime}=\eta_{1}=0$. La même convention de phases que celle de Shaffer et Louck a été adoptée dans les expressions des intensités rotationnelles de Townes et Schawlow [9].

1.2 REPRÉSENTATION COUPLÉE. - Les composantes $m$ de $\mathbf{j}$ et $n_{1}$ de $\mathbf{j}_{1}$ étant définies dans la représentation couplée $\left|j m j_{1} n_{1} j_{2}\right\rangle$, il faut déterminer, par une relation analogue à (3), deux angles de phases $\delta$ et $\delta_{1 \mathrm{c}}^{\prime}$. Ce dernier diffère de $\delta_{1}^{\prime}$, qui se rapporte aux variations de $n_{1}$ dans la représentation découplée. On peut calculer les éléments de matrice de $\mathbf{j}_{1}^{ \pm}$dans le référentiel où la composante quantifiée de $\mathbf{j}_{1}$ est $n_{1}$ par transformation dans la base découplée. Puisque cette transformation correspond au couplage (2) dans le référentiel où la composante quantifiée de $\mathbf{j}_{1}$ est $m_{1}$, les C. G. sont indépendants de $n_{1}$ et il vient

$$
\begin{aligned}
& \left\langle j m j_{1} n_{1} \pm 1 j_{2}\left|\mathbf{j}_{1}^{ \pm}\right| j m j_{1} n_{1} j_{2}\right\rangle=\sum_{\substack{m_{2} \\
m_{1}=m-m_{2}}} \\
& \times\left|\left\langle j_{1} m_{1} j_{2} m_{2} \mid j m j_{1} j_{2}\right\rangle\right|^{2} \\
& \times\left\langle j_{1} m_{1} n_{1} \pm 1\left|\mathbf{j}_{1}^{ \pm}\right| j_{1} m_{1} n_{1}\right\rangle .
\end{aligned}
$$

L'angle $\delta_{1 \mathrm{c}}^{\prime}$ est donc déterminé par l'angle $\delta_{1}^{\prime}$ de la représentation découplée :

$\delta_{1 \mathrm{c}}^{\prime}=\delta_{1}^{\prime}$.

L'effet de l'angle de phase $\delta$, qui se rapporte aux variations de $m$, sur la valeur des C. G. est analysé dans l'appendice I. Ici nous rappelons que, pour des valeurs de $\delta$ et $\delta_{1}$ données, il faut multiplier les C. G. standard par $\exp \left[i\left(\delta_{1}-\delta\right) m\right]$, et qu'il est commode de prendre $\delta=\delta_{1}$ afin de rendre les expressions des C. G. indépendantes de la valeur du paramètre $m$.

On peut définir les phases par rapport aux nombres quantiques $j$ et $j_{1}$ au moyen des angles $\eta$ et $\eta_{1 \mathrm{c}}$, tels que les éléments de matrice avec $\Delta j= \pm 1$ et ceux avec $\Delta j_{1}= \pm 1$ des composantes $z$ de $j_{1}$ et $\mathbf{j}$ non diagonales (c'est-à-dire $\mathbf{N}_{\boldsymbol{z}}$ et $\mathbf{J}_{\mathbf{z}}$ dans le cas du couplage entre la rotation et le spin électronique) soient donnés par des quantités réelles et positives multipliées respectivement par $\exp ( \pm i \eta)$ et $\exp \left( \pm i \eta_{1 \mathrm{c}}\right)$. Puisque les lignes des tableaux de $C$. G. correspondent aux différentes valeurs de $j-j_{1}$, et que $j_{1}$ est traité comme un paramètre, il est clair que les relations le long des colonnes dans les tableaux de C. G. sont déterminées par $\eta$. Dans les tableaux standard $\eta=0$. Alors, pour $\eta$ donné, il suffirait de multiplier les valeurs des C. G. standard par $\exp (-i \eta j)$; mais afin de rendre les expressions des C. G. qui correspondent à la même valeur de $j-j_{1}$ indépendantes de la valeur de $j_{1}$, il est préférable de multiplier les C. G. standard par $\exp \left[-i \eta\left(j-j_{1}\right)\right]$.

Les angles $\eta$ et $\eta_{1 \mathrm{c}}$ déterminent de même les facteurs de phase des éléments de matrice réduits des cosinus directeurs dans la représentation couplée avec $\Delta j= \pm 1$ et $\Delta j_{1}=0$ ou $\Delta j_{1}= \pm 1$ et $\Delta j=0$. On peut le démontrer aisément si on considère $\lambda_{z z}$ comme la composante $z$ d'un vecteur unitaire ayant pour direction $Z$. Au moyen du théorème de projection (Rose [2], page 94), on montre alors que les éléments de matrice de cet opérateur diagonaux en $j_{1}$ et ceux diagonaux en $j$, sont donnés respectivement par les éléments de matrice des opérateurs

$$
\mathbf{j}_{1 z} \mathbf{j}_{1 z} /\left[j_{1}\left(j_{1}+1\right)\right] \quad \text { et } \quad \mathbf{j}_{z} \mathbf{j}_{z} /[j(j+1)] \text {. }
$$


Puisque les éléments de matrice réduits de $\lambda$ dans les deux représentations couplée et découplée sont liés par la relation (II.5), cela signifie qu'il y a aussi une relation entre les angles $\eta, \eta_{1 \mathrm{c}}$, et $\eta_{1}$. Afin de trouver cette relation, on exprime $j_{z}$ en fonction de $\lambda_{00}=\lambda_{z z}$ par le théorème de projection déjà mentionné et, appliquant la relation (II.1) aux éléments de matrice de $\lambda_{00}$, on obtient :

$$
\begin{aligned}
& \left\langle j m j_{1} \pm 1 n_{1} j_{2}\left|\mathbf{j}_{z}\right| j m j_{1} n_{1} j_{2}\right\rangle=\frac{j(j+1)}{m} C(j 1 j ; m 0 m) C\left(j_{1} 1 j_{1} \pm 1 ; n_{1} 0 n_{1}\right) \times \\
& \quad \times\left\langle j j_{1} \pm 1 j_{2}\|\lambda\| j j_{1} j_{2}\right\rangle .
\end{aligned}
$$

Le premier C. G. dans l'équation (8) vaut $m /[j(j+1)]^{1 / 2}$, et appliquant la relation (II.5) on arrive au résultat :

$$
\begin{aligned}
& \left\langle j m j_{1} \pm 1 n_{1} j_{2}\left|\mathbf{j}_{z}\right| j m j_{1} n_{1} j_{2}\right\rangle=\exp (\mp i \eta)(2 j+1)[j(j+1)]^{1 / 2} C\left(j_{1} 1 j_{1} \pm 1 ; n_{1} 0 n_{1}\right) \times \\
& \quad \times\left[(-1)^{j_{2}+1-j_{1}-j} W\left(j_{1} \pm 1 j_{1} j j ; 1 j_{2}\right)\right]\left\langle j_{1} \pm 1\|\lambda\| j_{1}\right\rangle .
\end{aligned}
$$

Le terme de gauche contient par définition le facteur de phase $\exp \left( \pm i \eta_{1 \mathrm{c}}\right)$. A droite il y a un terme $\exp (\mp i \eta)$ et l'élément de matrice réduit contient le facteur de phase $\exp \left( \pm i \eta_{1}\right)$; de plus le C. G. et le terme entre crochets sont réels, respectivement avec le signe \pm sorte qu'ils donnent ensemble un facteur $\exp (i \pi)$. On obtient alors la relation

$\eta_{1 \mathrm{c}}=\eta_{1}-\eta+\pi$. et $\mp$ (voir les tableaux I.2 et I.4 de Rose [2]), de

1.3 CONCLUSIONS GÉNÉRALES. - En examinant le problème du couplage $\mathbf{j}=\mathbf{j}_{1}+\mathbf{j}_{2}$ entre la rotation moléculaire et le spin électronique $\left(\mathbf{j}_{2}\right)$ dans les deux paragraphes précédents, nous avons défini des angles de phases par rapport aux variations des nombres quantiques dans les représentations découplée et couplée. Le schéma suivant sert à rappeler les notations des angles de phases et des nombres quantiques correspondants :

$$
\begin{array}{lllllll}
j_{1} & m_{1} & n_{1} & j_{2} & m_{2} & j & m
\end{array}
$$

Représentation découplée

$\left|j_{1} m_{1} n_{1}\right\rangle\left|j_{2} m_{2}\right\rangle$

$\eta_{1} \quad \delta_{1} \quad \delta_{1}^{\prime} \quad-$

$\delta_{2}$

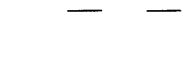

Représentation couplée

$\left|j m j_{1} n_{1} j_{2}\right\rangle$.

$$
\eta_{1 \mathrm{c}}-\delta_{1 \mathrm{c}}^{\prime}-\quad-\eta \delta
$$

Ces angles obéissent aux relations (7) et (10).

Puisque nous considérons seulement les interactions dans un état de multiplicité de spin déterminée, nous ne définissons aucun angle par rapport à $j_{2}$. Dans les tableaux standard de C. G. [1-3] $\delta_{1}=\delta_{2}$ et $\eta=0$. Avec des phases générales, si on veut que les expressions des C. G. soient indépendantes de la valeur de $m$ et de $j_{1}$, qui y apparaissent comme des paramètres, il suffit de multiplier les valeurs des C. G. standard par $\exp \left\{-i\left[\left(\delta_{1}-\delta_{2}\right) m_{2}+\eta\left(j-j_{1}\right)\right]\right\}$. Dans ce cas on a $\delta=\delta_{1}$. Il est commode de multiplier de plus par la constante $\exp \left[i\left(\delta_{1}-\delta_{2}+\eta\right) j_{2}\right]$, de façon que le C. G. avec $m_{1}=j_{1}, m_{2}=j_{2}$ et $j=j_{1}+j_{2}$ soit, selon l'usage, réel et positif dans tous les cas. Alors on obtient :

$$
\begin{aligned}
& \left\langle j_{1} m_{1} n_{1} j_{2} m_{2} \mid j m j_{1} n_{1} j_{2}\right\rangle= \\
& \quad=\exp \left\{-i\left[\left(\delta_{1}-\delta_{2}\right)\left(m_{2}-j_{2}\right)+\right.\right. \\
& \left.\left.\quad+\eta\left(j-j_{1}-j_{2}\right)\right]\right\} C\left(j_{1} j_{2} j ; m_{1} m_{2} m\right) .
\end{aligned}
$$

2. Exemples et applications. - 2.1 ReLATIONS RELATIVES AUX LIGNES. - Nous allons présenter ici des exemples où un choix de phases non conventionnel apporte des changements aux relations le long des lignes des C. G. Un cas très important est représenté par le couplage entre la rotation moléculaire et le spin électronique dans un système d'axes lié à la molécule $\mathbf{N}=\mathbf{J}+\tilde{\mathbf{S}}$, où $\tilde{\mathbf{S}}=-\mathbf{S}$. Pour garder les relations de phase conventionnelles, il faudrait que $\delta_{1}=\delta_{2}$, ces angles étant associés aux opérateurs $\mathbf{J}^{ \pm}$et $\widetilde{\mathbf{S}}^{ \pm}=-\mathbf{S}^{ \pm}$. Mais selon l'usage on prend les éléments de matrice de $\mathbf{J}^{ \pm}$et $\mathbf{S}^{ \pm}$réels et positifs, et alors ceux de $\widetilde{\mathbf{S}}^{ \pm}$deviennent négatifs. Cela signifie que $\delta_{1}=0$ et $\delta_{2}=\pi$, et par conséquent il faut changer de signe les colonnes paires ou impaires des tableaux de C. G. standard, suivant l'équation (11). C'est ce qui a été fait par Hougen dans son article sur les intensités rotationnelles des transitions singulet-triplet dans le cas b de couplage [10]. Il prend les éléments de matrice des cosinus directeurs comme Shaffer et Louck [8], 
où $\delta_{1}=\delta_{1}^{\prime}=\eta_{1}=0$, et il prend $\delta_{2}=\pi$ à cause des changements de signes mentionnés; les éléments de matrice de $\widetilde{\mathbf{S}}^{ \pm}=-\mathbf{S}^{ \pm}$sont donc réels et négatifs. Alors si on rencontre les éléments de matrice de $\mathbf{S}^{ \pm}$ dans un problème où l'on adopte les expressions d'intensité d'Hougen, il faudra les prendre réels et positifs. Ces mêmes considérations valent pour les formules des intensités rotationnelles de di Lauro pour les transitions doublet-quartet [11] et doubletdoublet [12]. Si on ne prend pas de facteurs de phase contenant $k$, les éléments de matrice de $\mathbf{N}^{ \pm}$dans la représentation couplée seront aussi réels et positifs, en accord avec la relation $\delta=\delta_{1}$.

Les phases déterminées par les angles $\delta_{1}, \delta_{2}$ ou $\delta$ dépendent aussi de la définition des opérateurs qui changent les nombres quantiques de projection. Par exemple, dans le référentiel mobile on prend selon l'usage général $\mathbf{N}^{ \pm}=\mathbf{N}_{\boldsymbol{x}} \mp i \mathbf{N}_{\boldsymbol{y}}$. Mais King, Hainer et Cross [13] jugèrent peu commode de faire correspondre la combinaison + au déplacement vers le bas et la combinaison - au déplacement vers le haut; c'est pourquoi ils ont défini $\mathbf{N}^{ \pm}=\mathbf{N}_{y} \pm i \mathbf{N}_{x}$, avec des éléments de matrice réels et positifs. Alors, suivant ces derniers auteurs, les éléments de matrice de $\mathbf{N}_{x} \mp i \mathbf{N}_{y}$ seraient imaginaires. Plusieurs auteurs, dont Posener et Strandberg [14], ont suivi King, Hainer et Cross.

Dans les problèmes concernant la structure rotationnelle des états multiplets, il est très important de comparer les éléments de matrice des opérateurs d'interaction spin-rotation et spin-spin de Van Vleck [4] à ceux de Raynes [15], puisque ces auteurs ont évidemment suivi des conventions de phases différentes. Dans le tableau des éléments de matrice de Van Vleck $\mathbf{N}^{ \pm}=\mathbf{N}_{x} \mp i \mathbf{N}_{y}$ et $\delta=0$. Raynes ne spécifie pas explicitement ses conventions, mais il se réfère à Posener et Strandberg [14] et son signe pour les éléments de matrice de l'opérateur $\mathbf{N}_{x}^{2}-\mathbf{N}_{y}^{2}$, avec $\Delta k= \pm 2$, est opposé au signe retenu par Van Vleck en accord avec le choix $\delta=0$ pour $\mathbf{N}^{ \pm}=\mathbf{N}_{y} \pm i \mathbf{N}_{x}$. Tous les autres éléments de matrice non diagonaux en $k$ de Raynes sont identiques à ceux de Van Vleck; alors on devrait penser que les composantes d'interaction spin-rotation et spin-spin de Raynes sont en général différentes de celles de Van Vleck. Brand, di Lauro et Liu [16] ont donné dans un tableau la correspondance entre les constantes de Van Vleck et celles de Raynes; cela explique bien la différence de signe entre la valeur de la constante de couplage spinspin $\beta$ pour l'état ${ }^{3} \mathrm{~B}_{1}$ de $\mathrm{SO}_{2}$ trouvée par Brand, Jones et di Lauro [17], qui l'ont calculée selon les éléments de matrice de Raynes, et la valeur trouvée par Tinti [18], qui a suivi Van Vleck. Hallin et al. [19] soutiennent que Raynes a malheureusement mélangé les phases de Posener et Strandberg et celles de Van Vleck, mais leur affirmation est arbitraire, car il est possible d'interpréter les éléments de matrice de Raynes conformément à la convention de Posener et Strandberg ou King, Hainer et Cross.

\subsection{Relations relatives auX COLONNes. - La} représentation couplée dans les problèmes concernant la rotation des molécules avec spin électronique peut être obtenue de deux façons : le couplage $\mathbf{N}=\mathbf{J}-\mathbf{S}$ dans le référentiel mobile et le couplage $\mathbf{J}=\mathbf{N}+\mathbf{S}$ dans le référentiel fixe. Les deux procédés donnent en général des représentations couplées avec des phases différentes, à moins qu'on ne choisisse correctement les valeurs des angles de phase pour les expressions des C. G. dans les deux transformations mentionnées. On rencontre cette situation dans le calcul des niveaux d'énergie et des intensités dans la structure rotationnelle des transitions entre états multiplets près du cas $b$, en présence d'un champ externe. Il est tout à fait correct d'écrire la matrice de l'énergie dans la représentation découplée $(\mathbf{N}, \mathbf{S})$ et d'évaluer les facteurs d'intensité dans la représentation découplée $(\mathbf{J}, \mathbf{S})$, et de transformer le tout dans la représentation couplée suivant les deux voies déjà mentionnées. Le schéma suivant nous donne les angles qui définissent les phases de la représentation couplée obtenue dans les deux cas, déterminés à $2 n \pi$ près :

$\begin{array}{llll}\text { Notation générale } & \eta & \eta_{1} & \eta_{1 \mathrm{c}}=\eta_{1}-\eta+\pi \\ \text { Couplage } \mathbf{J}=\mathbf{N}+\mathbf{S} & \eta_{\mathrm{Jc}} & \eta_{N} & \eta_{N_{\mathrm{c}}}=\eta_{N}-\eta_{J \mathrm{c}}+\pi \\ \text { Couplage } \mathbf{N}=\mathbf{J}-\mathbf{S} & \eta_{N \mathrm{c}} & \eta_{J} & \eta_{J \mathrm{c}}=\eta_{J}-\eta_{N \mathrm{c}}+\pi\end{array}$

Il faut que les angles correspondants désignés par les mêmes symboles dans $(12 a)$ et $(12 b)$ aient la même valeur pour obtenir la représentation couplée avec les mêmes phases par les deux procédés de couplage. A cette condition, tirant $(12 b)$ de $(12 a)$ on obtient :

$\eta_{N}=\eta_{J}$

Suivant les relations relatives aux colonnes des C. G. standard, $\eta_{\mathrm{Jc}}=\eta_{\mathrm{Nc}}=0$, et en considérant les équations $(12 a, b)$ il vient :
$\eta_{N}=\eta_{J}=\pi$.

Cela signifie que les éléments matriciels réduits non diagonaux des cosinus directeurs, donnés par l'équation (5), sont négatifs dans les deux représentations découplées et alors qu'il faudrait changer de signe tous les éléments de matrice avec $\Delta j_{1}= \pm 1$ de Shaffer et Louck [8] ou Townes et Schawlow [9], où $\eta_{1}=0$, dans le calcul des intensités.

Buckingham et al., dans un travail sur l'effet Stark 
dans la transition ${ }^{3} \mathrm{~A}_{2}-{ }^{1} \mathrm{~A}_{1}$ de la formaldéhyde [20], ont fait un choix différent : pour garder les expressions des intensités d'Hougen [10], où $\eta_{N c}=0$ et $\eta_{J}=\eta_{N}=0$, ils changent de signe toutes les lignes paires ou impaires des C. G. pour le couplage $\mathbf{J}=\mathbf{N}+\mathbf{S}$ dans le référentiel fixe. Cela revient à prendre $\eta_{J \mathrm{c}}=\pi$, en accord avec $(12 a, b)$ sous la condition $\eta_{J}-\eta_{N \mathrm{c}}=\eta_{N}-\eta_{N \mathrm{c}}=0$.

\section{Appendice I}

Une relation relative aux lignes des $\mathrm{C}$. G. et le théorème de Wigner-Eckart. $-\mathrm{Si}$ on prend des phases arbitraires, la relation (5.18) de Rose [2] entre les C. G. correspondant aux valeurs successives de $m$ pour le couplage $\mathbf{j}=\mathbf{j}_{1}+\mathbf{j}_{2}$ devient :

$$
\begin{aligned}
\exp & \pm i \delta)[j(j+1)-m(m \pm 1)]^{1 / 2}\left\langle j_{1} m_{1} j_{2} m_{2} \mid j m \pm 1 j_{1} j_{2}\right\rangle= \\
& =\exp \left( \pm i \delta_{1}\right)\left[j_{1}\left(j_{1}+1\right)-\left(m_{1} \mp 1\right) m_{1}\right]^{1 / 2}\left\langle j_{1} m_{1} \mp 1 j_{2} m_{2} \mid j m j_{1} j_{2}\right\rangle+ \\
& +\exp \left( \pm i \delta_{2}\right)\left[j_{2}\left(j_{2}+1\right)-\left(m_{2} \mp 1\right) m_{2}\right]^{1 / 2}\left\langle j_{1} m_{1} j_{2} m_{2} \mp 1 \mid j m j_{1} j_{2}\right\rangle .
\end{aligned}
$$

Après multiplication $\operatorname{par}\left\langle j m j_{1} j_{2} \mid j_{1} m_{1} \mp 1 j_{2} m_{2}\right\rangle$ et sommation sur $j$, on obtient au moyen d'une relation d'orthonormalité des C. G. :

$$
\begin{aligned}
& \exp ( \pm i \delta) \sum_{j}[j(j+1)-m(m \pm 1)]^{1 / 2}\left\langle j_{1} m_{1} j_{2} m_{2} \mid j m \pm 1 j_{1} j_{2}\right\rangle\left\langle j m j_{1} j_{2} \mid j_{1} m_{1} \mp 1 j_{2} m_{2}\right\rangle= \\
& \quad=\exp \left( \pm i \delta_{1}\right)\left[j_{1}\left(j_{1}+1\right)-\left(m_{1} \mp 1\right) m_{1}\right]^{1 / 2} .
\end{aligned}
$$

Divisant (I.2) par exp $\left( \pm i \delta_{1}\right)$, on fait apparaitre dans le terme de gauche un facteur $\exp \left[ \pm i\left(\delta-\delta_{1}\right)\right]$, tandis que le terme de droite devient réel et positif. Cela signifie que les produits

$$
\left\langle j_{1} m_{1} j_{2} m_{2} \mid j m \pm 1 j_{1} j_{2}\right\rangle\left\langle j m j_{1} j_{2} \mid j_{1} m_{1} \mp 1 j_{2} m_{2}\right\rangle
$$

entre C. G. différant seulement par une unité en $m$ contiennent un facteur $\exp \left[i\left(\delta_{1}-\delta_{2}\right) m\right]$. Le nombre quantique $m$ étant traité comme un paramètre dans les tableaux de C. G., si on veut que les expressions des C. G. soient indépendantes de la valeur de $m$, il faut choisir $\delta=\delta_{1}$. L'équation (I.1) est importante même dans le procédé de dérivation du théorème de Wigner-Eckart. On définit selon l'usage les composantes sphériques $\mathbf{T}_{q}^{(t)}$ d'un tenseur irréductible $\mathbf{T}$ de rang $t$ par rapport à une base de moment cinétique $|J M\rangle$ par les relations de commutations

$$
\begin{aligned}
& {\left[\mathbf{J}_{z}, \mathbf{T}_{q}^{(t)}\right]=q \mathbf{T}_{q}^{(t)}} \\
& {\left[\mathbf{J}^{ \pm}, \mathbf{T}_{q}^{(t)}\right]=[t(t+1)-q(q \pm 1)]^{1 / 2} \mathbf{T}_{q \pm 1}^{(t)}}
\end{aligned}
$$

où les indices supérieurs de $\mathbf{J}^{ \pm}$identifient les opérateurs qui modifient le nombre quantique de projection de $\mathbf{J}$ de \pm 1 .

Avec un angle de phase $\delta_{J}$ arbitraire pour $J^{ \pm}$, suivant le procédé de Rose [2] (pp. 85-88) on obtient la relation :

$$
\begin{aligned}
& \exp \left(\mp i \delta_{J}\right)\left[J^{\prime}\left(J^{\prime}+1\right)-M^{\prime}\left(M^{\prime} \pm 1\right)\right]^{1 / 2}\left\langle J^{\prime} M^{\prime} \pm 1\left|\mathbf{T}_{q}^{(t)}\right| J M\right\rangle= \\
& \quad=\exp \left(\mp i \delta_{J}\right)[J(J+1)-M(M \mp 1)]^{1 / 2}\left\langle J^{\prime} M^{\prime}\left|\mathbf{T}_{q}^{(t)}\right| J M \mp 1\right\rangle+ \\
& \quad+[t(t+1)-q(q \mp 1)]^{1 / 2}\left\langle J^{\prime} M^{\prime}\left|\mathbf{T}_{q \mp 1}^{(t)}\right| J M\right\rangle
\end{aligned}
$$

(I.5) est similaire au complexe conjugué de (I.1), si on fait correspondre $J, M, t, q, J^{\prime}, M^{\prime}$, à $j_{1}, m_{1}, j_{2}, m_{2}$, $j, m$, et les éléments de matrice dans (I.5) aux complexes conjugués des C. G. dans (I.1), à condition que $\delta_{J}=\delta=\delta_{1}$ et $\delta_{2}=0$. Ceci signifie que les expressions des éléments matriciels des opérateurs $\mathbf{T}_{q}^{(t)}$ dans la base $|J M\rangle$ obtenus par le théorème de Wigner-Eckart contiennent les complexes conjugués de $\mathrm{C}$. $\mathrm{G}$. du type $\left\langle J^{\prime} M+q J t \mid J M t q\right\rangle$. Puisque la valeur des C.G. dépend de $\delta_{1}-\delta_{2}$ selon l'équation (11), si on indique les C. G. des tableaux standard par les symboles $C$ de Rose et on tient compte des conditions $\delta_{J}=\delta_{1}$ et $\delta_{2}=0$ mentionnées ci-dessus, on obtient :

$\left\langle J^{\prime} M+q\left|\mathbf{T}_{q}^{(t)}\right| J M\right\rangle=\exp \left(i \delta_{J} q\right) C\left(J t J^{\prime} ; M q M+q\right)\left\langle J^{\prime}\|\mathbf{T}\| J\right\rangle$. 
Il faut remarquer que le terme $\exp \left(i \delta_{J} q\right)$ dans (I.6) tient compte des phases des fonctions $|J M\rangle$ relativement au nombre quantique de projection $M$, le facteur de phase relatif au nombre quantique $J$ étant incorporé dans la valeur de l'élément de matrice réduit $\left\langle J^{\prime}\|\mathbf{T}\| J\right\rangle$. Si les composantes cartésiennes de $\mathbf{T}^{(t)}$ sont hermitiques, alors $\left(\mathbf{T}_{q}^{(t)}\right)^{+}=(-1)^{q} \mathbf{T}_{-q}^{(t)}$. Lorsqu'on applique cette relation à (I.6), il vient [21] :

$\left\langle J^{\prime}\|\mathbf{T}\| J\right\rangle=(-1)^{J^{\prime}-J}\left(\frac{2 J+1}{2 J^{\prime}+1}\right)^{1 / 2}\left\langle J\|\mathbf{T}\| J^{\prime}\right\rangle^{*}$.

\section{Appendice II}

Opérateurs cosinus directeurs. - Chaque cosinus directeur $\lambda_{F g}$ représente soit la composante cartésienne $F$ dans le référentiel fixe d'un vecteur unitaire ayant pour direction $g$, soit la composante $g$ dans le référentiel mobile d'un vecteur unitaire ayant pour direction $F$. Shaffer et Louck [8] ont transformé les cosinus directeurs en opérateurs $\lambda_{\mu \nu}$ qui ont les propriétés des composantes sphériques dans les deux référentiels et dans toute base de moments cinétiques qui contiennent la rotation moléculaire. Alors on peut évaluer leurs éléments de matrice dans la base $\left|j_{1} m_{1} n_{1}\right\rangle$ en utilisant le théorème de Wigner-Eckart (voir appendice I et en particulier l'équation (I.6)), et on obtient

$$
\begin{aligned}
& \left\langle j_{1}^{\prime} m_{1}+\mu n_{1}+v\left|\lambda_{\mu v}\right| j_{1} m_{1} n_{1}\right\rangle=\exp \left[i\left(\delta_{1} \mu+\delta_{1}^{\prime} v\right)\right] C\left(j_{1} 1 j_{1}^{\prime} ; m_{1} \mu m_{1}+\mu\right) \times \\
& \times C\left(j_{1} 1 j_{1}^{\prime} ; n_{1} v n_{1}+v\right)\left\langle j_{1}^{\prime}\|\lambda\| j_{1}\right\rangle .
\end{aligned}
$$

Dans cette équation $j_{1}^{\prime}=j_{1}, j_{1}+1, j_{1}-1$ et les angles $\delta_{1}$ et $\delta_{1}^{\prime}$ sont déterminés par les phases des fonctions $\left|j m_{1} n_{1}\right\rangle$ par rapport aux variations des nombres quantiques $m_{1}$ et $n_{1}$ respectivement. Par contre, le facteur de phase par rapport aux variations de $j_{1}$ est incorporé dans la valeur de l'élément de matrice réduit. L'équation (II.1) diffère de (I.6) puisqu'elle contient deux C. G. correspondant au couplage formel $j_{1}+1=j_{1}^{\prime}$. Par conséquent, l'analogue de (I.7) pour les cosinus directeurs est

$$
\left\langle j_{1}^{\prime}\|\lambda\| j_{1}\right\rangle=\left[\left(2 j_{1}+1\right) /\left(2 j_{1}^{\prime}+1\right)\right]\left\langle j_{1}\|\lambda\| j_{1}^{\prime}\right\rangle^{*} \text {. }
$$

Dans la représentation couplée on obtient :

$$
\begin{aligned}
& \left\langle j^{\prime} m+\mu j_{1}^{\prime} n_{1}+v j_{2}\left|\lambda_{\mu v}\right| j m j_{1} n_{1} j_{2}\right\rangle=\exp \left[i\left(\delta \mu+\delta_{1 \mathrm{c}}^{\prime} v\right)\right] C\left(j 1 j^{\prime} ; m \mu m+\mu\right) \times \\
& \quad \times C\left(j_{1} 1 j_{1}^{\prime} ; n_{1} v n_{1}+v\right)\left\langle j^{\prime} j_{1}^{\prime} j_{2}\|\lambda\| j j_{1} j_{2}\right\rangle . \\
& \left\langle j^{\prime} j_{1}^{\prime} j_{2}\|\lambda\| j j_{1} j_{2}\right\rangle=(-1)^{j^{\prime}-j+j_{1}-j_{1}}\left[\frac{(2 j+1)\left(2 j_{1}+1\right)}{\left(2 j^{\prime}+1\right)\left(2 j_{1}^{\prime}+1\right)}\right]^{1 / 2}\left\langle j j_{1} j_{2}\|\lambda\| j^{\prime} j_{1}^{\prime} j_{2}\right\rangle^{*} .
\end{aligned}
$$

On peut aussi évaluer l'élément de matrice (II.3) en passant dans la base découplée, afin d'obtenir une relation entre les éléments de matrice réduits dans les deux représentations. Avec des phases générales on obtient :

$$
\begin{aligned}
& \left\langle j^{\prime} j_{1}^{\prime} j_{2}\|\lambda\| j j_{1} j_{2}\right\rangle=\exp \left\{i\left[\left(\delta_{1}-\delta\right) \mu+\left(\delta_{1}^{\prime}-\delta_{1 \mathrm{c}}^{\prime}\right) v\right]\right\} \exp \left[i \eta\left(j^{\prime}-j-j_{1}^{\prime}+j_{1}\right)\right] \times \\
& \times(-1)^{j_{2}+1-j_{1}-j^{\prime}}\left[\left(2 j^{\prime}+1\right)(2 j+1)\right]^{1 / 2} W\left(j_{1}^{\prime} j_{1} j^{\prime} j ; 1 j_{2}\right)\left\langle j_{1}^{\prime}\|\lambda\| j_{1}\right\rangle .
\end{aligned}
$$

Le symbole $W$ dans (II.5) est un coefficient de Racah standard. Cette équation diffère de l'équation (6.25) de Rose [2], qui se rapporte à des phases standard, par les deux termes exponentiels dont le premier, dépendant de la composante $\lambda_{\mu \nu}$, disparaît à cause de la relation (7) et du choix $\delta=\delta_{1}$.

\section{Bibliographie}

[1] Condon, E. U. and Shortley, G. H., The Theory of Atomic Spectra (Cambridge University Press, Cambridge, England) 1951.

[2] Rose, M. E., Elementary Theory of Angular Momentum (Wiley, New York) 1957.

[3] Wigner, E. P., Group Theory (Academic Press, New York) 1959.
[4] Van Vleck, J. H., Rev. Mod. Phys. 23 (1951) 213.

[5] Féménias, J. L. et Athénour, C., Can. J. Phys. 53 (1975) 533.

[6] Freed, K. F., J. Chem. Phys. 45 (1966) 4214.

[7] Brown, J. M. and Howard, B. J., Mol. Phys. 31 (1976) 1517.

[8] ShafFer, W. H. and Louck, J. D., J. Mol. Spectros. 3 (1959) 123. 
[9] Townes, C. H. and Schawlow, A. L., Microwave Spectroscopy (McGraw Hill, New York) 1955.

[10] Hougen, J. T., Can. J. Phys. 42 (1964) 433.

[11] Di Lauro, C., J. Mol. Spectros. 51 (1974) 50.

[12] Di Lauro, C., J. Mol. Spectros. 51 (1974) 356.

[13] King, G. W., Hainer, R. M. and Cross, P. C., J. Chem. Phys. 11 (1943) 27.

[14] Posener, D. W. and Strandberg, M. W. P., Phys. Rev. 95 (1954) 374 ; ibid. 96 (1954) 1714.

[15] Raynes, W. T., J. Chem. Phys. 41 (1964) 3020.
[16] Brand, J. C. D., di Lauro, C. and Liu, D. S., Can. J. Phys. 53 (1975) 1853.

[17] Brand, J. C. D., Jones, V. T. and di Lauro, C., J. Mol. Spectros. 40 (1971) 616.

[18] Tinti, D. S., Chem. Phys. Lett. 12 (1971) 169.

[19] Hallin, K. E. J., Hamada, Y. and Merer, A. J., Can. J. Phys. 54 (1976) 211.

[20] Buckingham, A. D., Ramsay, D. A. and Tyrrel, J., Can. J. Phys. 48 (1970) 1242.

[21] RacAH, G., Phys. Rev. 62 (1964) 438. 